\title{
In vitro Effects of Beet Root Juice on Stimulated and Unstimulated Peripheral Blood Mononuclear Cells
}

\author{
${ }^{1}$ Christiana Winkler, ${ }^{1}$ Barbara Wirleitner, ${ }^{1}$ Katharina Schroecksnadel \\ ${ }^{2}$ Harald Schennach and ${ }^{1}$ Dietmar Fuchs \\ ${ }^{1}$ Division of Biological Chemistry, Biocentre, Innsbruck Medical University, \\ Ludwig Boltzmann Institute of AIDS-Research, Innsbruck \\ ${ }^{2}$ Central Institute of Blood Transfusion and Immunology, University Hospital
}

\begin{abstract}
Intake of fruits and vegetables rich in antioxidants is suggested to reduce the incidence of cancer and coronary heart disease in humans. Exceptional antioxidant activity of beet root extracts has been reported. Likewise in animal models, e.g., extracts of red beetroot Beta vulgaris var. rubra revealed significant tumor inhibitory effects. Red beetroot concentrate is universally permitted as a food ingredient. In this study, effects of a commercially available beetroot juice on freshly isolated human peripheral blood mononuclear cells stimulated with the mitogens phytohaemagglutinin and concanavalin A were investigated in vitro. Tryptophan degradation and neopterin formation were monitored in culture supernatants to determine effects of test substances on immunobiochemical pathways which both are induced by the pro-inflammatory cytokine interferon- $\gamma$. Compared to unstimulated cells, the mitogens induced significant formation of neopterin and degradation of tryptophan which is reflected by increasing concentrations of kynurenine together with diminished tryptophan levels in supernatants. Addition of beetroot extracts significantly suppressed these mitogeninduced changes, e.g. the rate of neopterin production as well as tryptophan degradation was dosedependently suppressed. Our data show that beetroot extract is able to counteract pro-inflammatory cascades in peripheral blood mononuclear cells. Because inflammation is strongly involved in the development and progression of several clinical conditions including coronary heart disease and cancer, beneficial effect of beetroot extract may relate to this anti-inflammatory capacity.
\end{abstract}

Key words: Beetroot, tryptophan, kynurenine, indoleamine (2, 3)-dioxygenase, neopterin, inflammation

\section{INTRODUCTION}

Intake of fruits and vegetables rich in antioxidants is suggested to reduce the incidence of cancer and coronary heart disease in humans. In the general public, beet root juice is considered powerful to prevent infectious and malignant disease and indeed also scientific literature is existing which seems to support these considerations. In vitro inhibitory effect of Beta vulgaris root extract on Epstein-Barr virus early antigen induction was demonstrated using Raji cells ${ }^{[1]}$ and also in vivo extracts of Beta vulgaris var. rubra revealed significant tumor inhibitory effects in murine skin and lung cancer ${ }^{[1]}$. These findings suggest that beetroot ingestion can be a useful means to prevent development and progression of cancer. But extracts of beetroot also showed some antimicrobial activity on Staphylococcus aureus and on Escherichia coli and also antiviral effect was observed ${ }^{[2,3]}$. Such data agree with a large number of different antimicrobial compounds, like phytoalexins, found in nature that play an important role in the natural defence of all kinds of living organisms ${ }^{[2]}$.
In the pathogenesis of various diseases, oxidative stress plays an important role. Reactive Oxygen Species (ROS), especially superoxide anion and hydrogen peroxide, are important signalling molecules in developing and proliferating cells but also in the induction of programmed cell death (apoptosis) ${ }^{[4]}$. ROS also participate in the expression of pro-inflammatory phenotypes and in the modification of the extracellular matrix $^{[5]}$. Diseases, like cancer, but also HIV-infection and atherosclerosis go along with immune activation which leads to increased production of ROS and oxidative stress may develop when antioxidant pools are overwhelmed. Such clinical conditions coincide with higher production of the immune activation marker neopterin ${ }^{[6]}$ and the accelerated degradation of essential amino acid tryptophan ${ }^{[7]}$. Increased amounts of neopterin are produced by human monocyte-derived macrophages upon stimulation with Th1-type cytokine interferon- $\gamma^{[6]}$. Interferon- $\gamma$ is also the most important trigger of the production of $\operatorname{ROS}^{[8]}$ and also stimulates enzyme Indoleamine (2, 3)-Dioxygenase (IDO) which converts tryptophan into kynurenine ${ }^{[7]}$. Higher concentrations of neopterin and accelerated degradation 
of tryptophan were found to predict disease progression and shorter survival expectation in cancer patients ${ }^{[9-12]}$.

In this study, we investigated the influence of red beetroot juice on stimulated and unstimulated PBMC of healthy donors by measurements of tryptophan degradation, kynurenine and neopterin concentrations.

\section{MATERIALS AND METHODS}

Test substance: A commercially available pure juice of biological cultivated beetroot (Bio-Randensaft aus biologischem Anbau, Biotta Ag, Tägerwilen, Switzerland) was used.

Isolation of human PBMC: PBMC were isolated from whole blood obtained from healthy voluntary donors. Separation of blood cells was performed using density centrifugation (Lymphoprep, Nycomed Pharma AS, Oslo, Norway). After isolation, PBMC were washed three times in phosphate buffered saline containing $0.2 \% 0.5 \mathrm{mM}$ EDTA. Cells were maintained in RPMI 1640 supplemented with $10 \%$ heat-inactivated fetal calf serum (Biochrom, Berlin, Germany), 1\% of $200 \mathrm{mM}$ glutamine (Serva, Heidelberg, Germany) and $0.1 \%$ of gentamycine $\quad\left(50 \mathrm{mg} \mathrm{mL}^{-1}\right.$, Bio-Whittaker, Walkersville, MD) in a humidified atmosphere containing $5 \% \mathrm{CO}_{2}$ for $48 \mathrm{~h}^{[13,14]}$. For each of the five experiments, PBMC were freshly prepared and for each two parallels were performed.

Stimulation of PBMC: Isolated PBMC were plated at a density of $1.5 \times 10^{6}$ cells $\mathrm{mL}^{-1}$ in supplemented RPMI 1640 , preincubated for $30 \mathrm{~min}$ with or without the beetroot extract $(0.1 \%, 1 \%$ and $5 \%)$ and stimulated or not with $10 \mu \mathrm{g} \mathrm{mL}^{-1}$ phytohaemagglutinin (PHA, Sigma, Vienna, Austria) and $10 \mu \mathrm{g} \mathrm{mL}^{-1}$ concanavalin A (Con A) for $48 \mathrm{~h}$.

Measurement of tryptophan degradation and of neopterin concentrations: After incubation, supernatants were harvested and tryptophan and kynurenine concentrations were measured by high pressure liquid chromatography (HPLC) using 3-nitroL-tyrosine as internal standard ${ }^{[15]}$. To estimate IDO activity, the kynurenine to tryptophan ratio (kyn/trp) was calculated and expressed as $\mu \mathrm{mol}$ kynurenine/ mmol tryptophan. Neopterin concentrations were determined by ELISA (BRAHMS Diagnostica, Berlin, Germany) according to the manufacturer's instructions with a detection limit of $2 \mathrm{nmol} \mathrm{L} \mathrm{L}^{-1}$.

Statistics: Data are presented as mean \pm SEM. To take inter-individual differences into account, for neopterin concentration results are presented as fold of unstimulated control of each experiment. For comparison of grouped data Mann-Whitney U-test was applied. p-values below 0.05 were considered to indicate significant differences.

\section{RESULTS}

Unstimulated cells: In supernatants of unstimulated PBMC average concentrations of tryptophan and kynurenine were mean \pm SEM: $17.0 \pm 2.0 \mu \mathrm{mol} \mathrm{L} \mathrm{L}^{-1}$ and $3.4 \pm 0.7 \mu \mathrm{mol} \mathrm{L}{ }^{-1}$, respectively. Kyn/trp was $291 \pm 96$ $\mu \mathrm{mol} \mathrm{mmol}{ }^{-1}$. The addition of the beetroot extract to the unstimulated PBMC suppressed tryptophan degradation: Kynurenine concentration and kyn/trp decreased and tryptophan concentrations increased at the two higher doses of $1 \%$ and $5 \%$ (at 5\%: tryptophan $28.6 \pm 0.8 \mu \mathrm{mol} \mathrm{L} \mathrm{L}^{-1}$, kynurenine $1.6 \pm 0.4 \mu \mathrm{mol} \mathrm{L} \mathrm{L}^{-1}$,

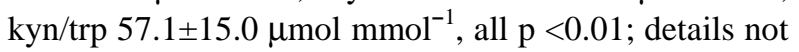
shown).

In unstimulated cells, neopterin concentration of $13.5 \mathrm{nmol} \mathrm{L}{ }^{-1} \pm 4.2$ was found. The addition of beetroot juice was able to suppress neopterin production to about $50 \%$ of baseline (average $7.6 \mathrm{nmol} \mathrm{L}^{-1}$ ).

Mitogen-stimulated cells: In stimulated PBMC activity of IDO was increased compared with unstimulated PBMC: In cells treated with PHA, a

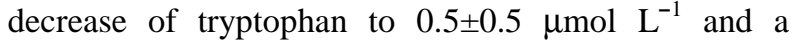
parallel increase of kynurenine concentrations to $9.5 \pm 0.3 \mu \mathrm{mol} \mathrm{L} \mathrm{L}^{-1}$ was observed (Fig. $1 \mathrm{a}$ and $1 \mathrm{~b}$ ). Accordingly, kyn/trp was more than 500-fold higher compared to unstimulated cells (Fig. 2a). Stimulation of cells with Con A decreased tryptophan concentration to $0.4 \pm 0.1 \mu \mathrm{mol} \mathrm{L}^{-1}$ and increased kynurenine concentration to $11.7 \pm 1.2 \mu \mathrm{mol} \mathrm{L}{ }^{-1}$ (Fig. $1 \mathrm{c}$ and d) and a nearly 150-fold higher kyn/trp was observed (Fig. 2b). Beet root juice inhibited stimulation-induced tryptophan degradation: kynurenine concentrations in supernatants were lower (Fig. 1a and b) and tryptophan concentrations were higher (Fig. 1c and d) in PBMC cultures treated with PHA or Con A plus beet root extract than in cultures with PHA or Con A only (Fig. 1a and b). Likewise, kyn/trp declined dose-dependently when extracts were added in the two higher concentrations (Fig. 2a and b). At 5\% concentration of extracts, kyn/trp reached values even below that of unstimulated cells.

In stimulated PBMC, mean neopterin concentration was $32 \mathrm{nmol} \mathrm{L}^{-1}$ for both mitogens (SEM \pm 5.2 for PHA and \pm 5.1 for Con A). In PHA stimulated cultures, the addition of $1 \%$ juice diminished neopterin production significantly. In PBMC stimulated with Con A, there was no difference in neopterin concentrations between the controls and the cells treated with $0.1 \%$. However, when cells were treated with the two higher, 1 and $5 \%$ concentrations of juice, significantly lower neopterin concentrations were obtained compared to cells which were exposed to $0.1 \%$ juice (Fig. $3 b$ ). 

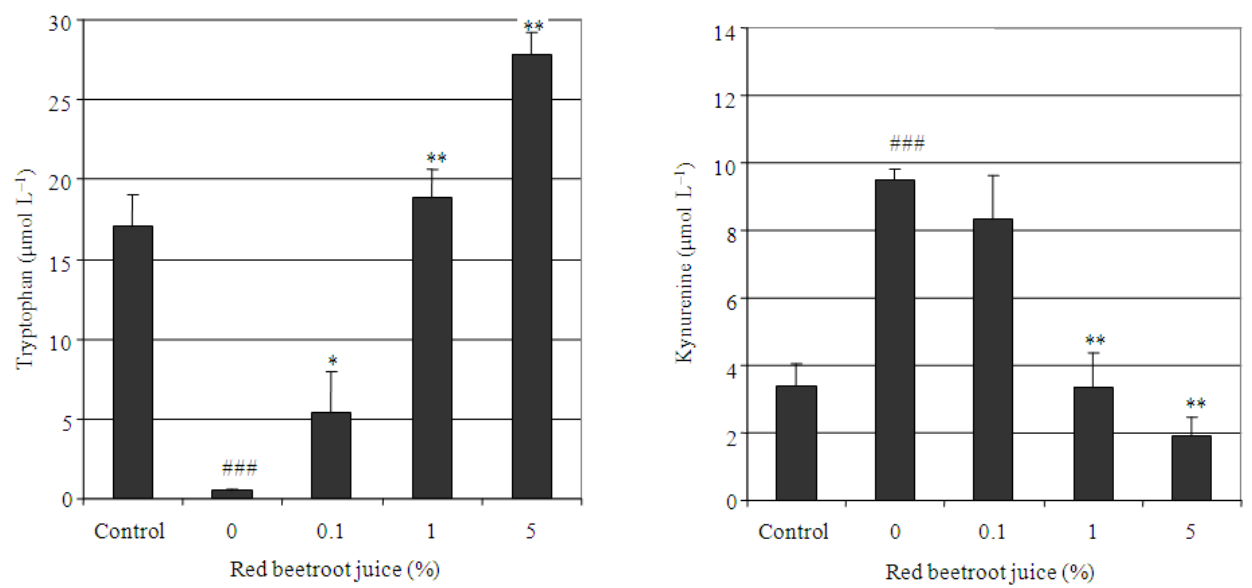

(a)
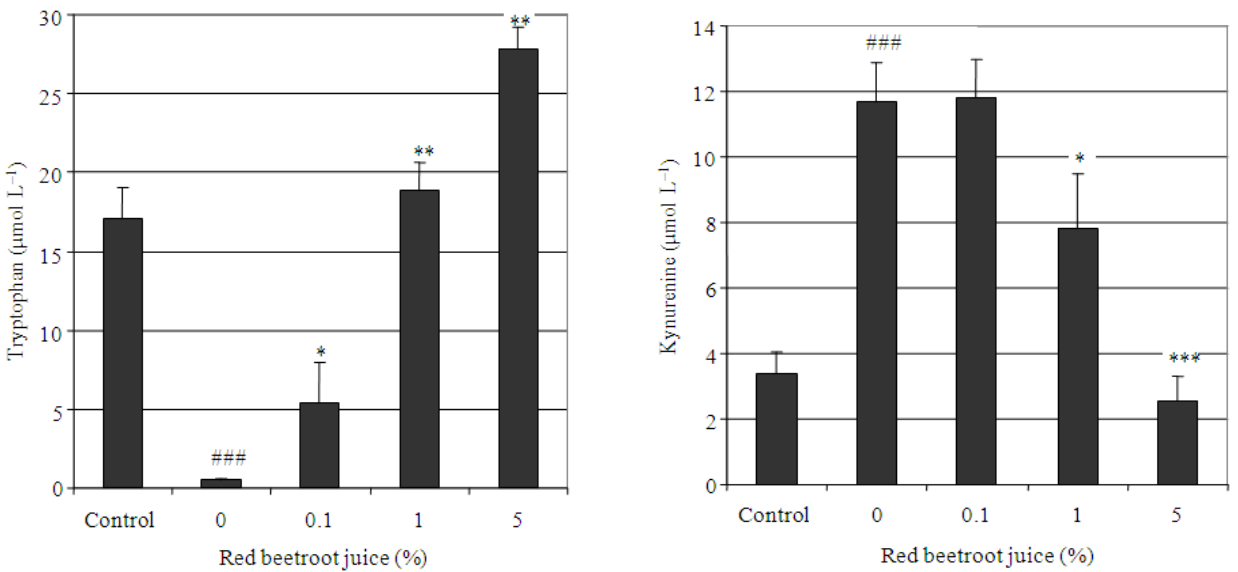

(b)

Fig. 1: Tryptophan concentrations (upper panel) and kynurenine concentrations (lower panel) in peripheral blood mononuclear cells, resting (control) or stimulated with phytohaemagglutinin (a) and concanavalin A (b) and coincubated with 3 different concentrations of red beet root juice for $48 \mathrm{~h}$, ${ }^{\# \# \#}$ : $\mathrm{p}<0.001$ compared to unstimulated control; *: $\mathrm{p}<0.05, * *: \mathrm{p}<0.001, * * *: \mathrm{p}<0.001$ compared to stimulated cells not treated with beet root juice $(0 \%)$

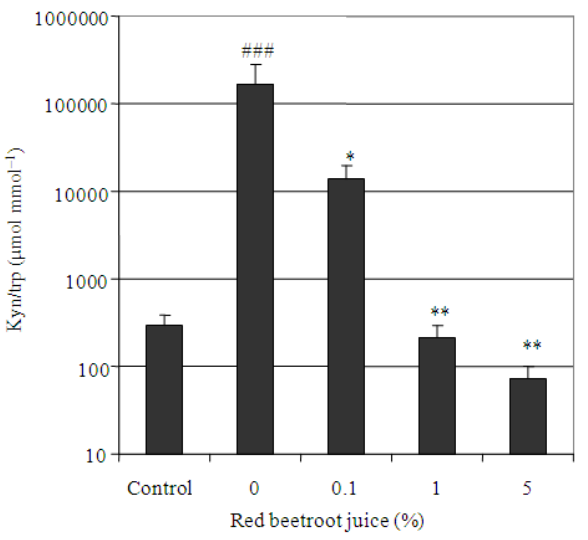

(a)

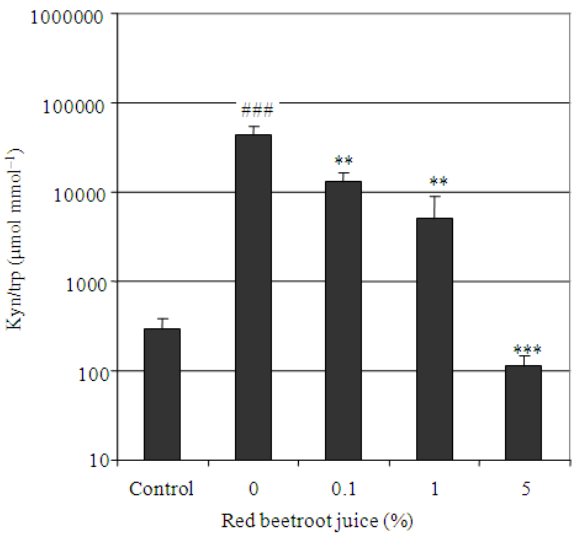

(b)

Fig. 2: Kynurenine to tryptophan ratio (Kyn/trp) in peripheral blood mononuclear cells resting (control) or stimulated with phytohaemagglutinin (a) and concanavalin A (b) and coincubated with 3 different concentrations of beet root juice for $48 \mathrm{~h},{ }^{\# \# \#: ~} \mathrm{p}<0.001$ compared to unstimulated control; *: $\mathrm{p}<0.05, * *$ : $\mathrm{p}<0.001, * * *: \mathrm{p}<0.001$ compared to stimulated cells not treated with beet root juice $(0 \%)$ 


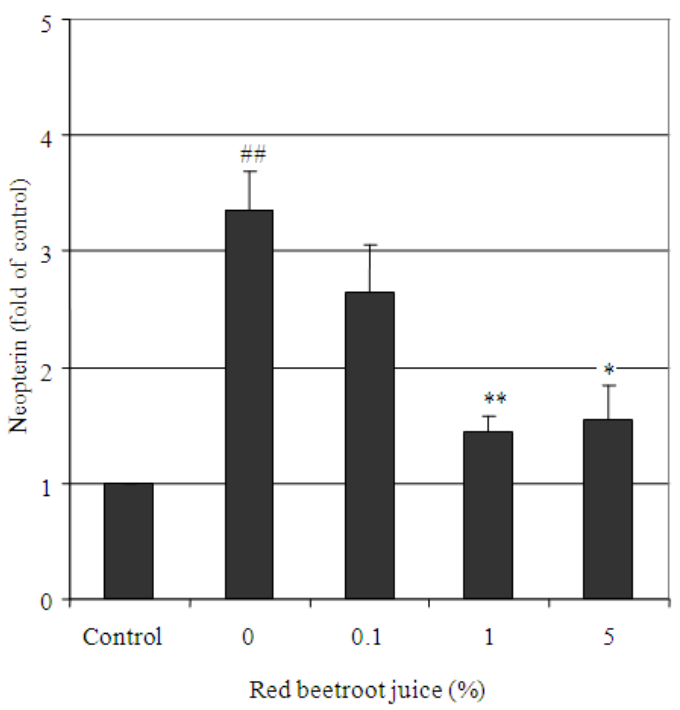

(a)

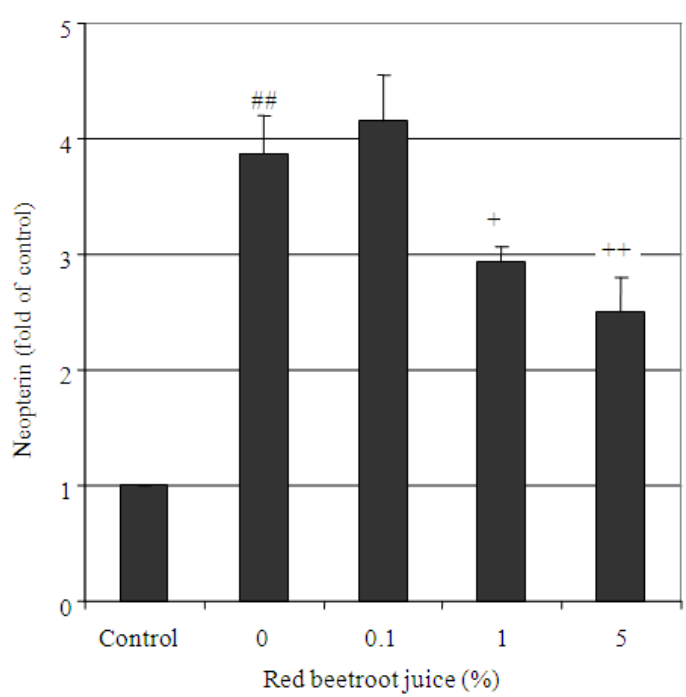

(b)

Fig. 3: Neopterin formation (fold of control $=1$ ) in peripheral blood mononuclear cells, resting (control) or stimulated with phytohaemagglutinin (a) and concanavalin A (b) and coincubated with 3 different concentrations of beet root juice for $48 \mathrm{~h},{ }^{\#}$ : $\mathrm{p}<0.01$, compared to unstimulated control; *: $\mathrm{p}<0.05$, **: $\mathrm{p}<0.001$, compared to stimulated cells not treated with beet root juice $(0 \%) ;{ }^{+}: \mathrm{p}<0.05,{ }^{++}: \mathrm{p}<0.001$ compared to cells treated with $0.1 \%$ beet root juice

\section{DISCUSSION}

Beetroot extract was found to dose-dependently suppress degradation of tryptophan and production of neopterin in PBMC which were stimulated with mitogens PHA and Con A. Because both immunobiological pathways are induced by the proinflammatory cytokine interferon- $\gamma^{[16,17]}$, data suggests a suppressive effect of the test substance on the formation and release of cytokine interferon- $\gamma$. Interferon- $\gamma$ was found earlier to be increased in PBMC stimulated with mitogens ${ }^{[14,18]}$ and induces the degradation of tryptophan and production of neopterin in mitogenstimulated PBMC.

Antioxidant compounds of beta vulgaris could be responsible for the influence of extracts on stimulated PBMC, because similar suppressive effects were observed earlier not only when using anti-inflammatory cytokines $^{[18]}$, but also with green and black te ${ }^{[19]}$, with extracts of Uncaria tomentosa ${ }^{[15]}$ or with potent antioxidant resveratrol ${ }^{[20]}$. Data imply that antioxidant compounds may not only suppress production of Th1type cytokine interferon- $\gamma$, moreover, in the sense of a cross-regulatory interplay between Th1-type and Th2type immune response, they may increase the susceptibility for allergic reactions when exposed to an allergen $^{[21]}$. This appears to be of special importance regarding beet root juice, because beetroot's red pigment betalain is widely used in food industry as a colorant.

Comparing 22 vegetable extracts revealed that beet after beans contains highest total phenol content per fresh weight and is among the 10 most potent antioxidant vegetables ${ }^{[22]}$. Compared to berries, beetroot peel extract had very low phenol contents, but despite that, showed remarkable antioxidant activity ${ }^{[23]}$.

Different beet root compounds, e.g., betalains, became especially important for phytomedicine: betalains (betacyanins and betaxanthis) have been detected only in red-violet-, orange-and yellow-pigmented botanical species belonging to closely related families of the order caryophyllalles ${ }^{[24]}$. Betalain pigments have specifically been shown to possess various antioxidant functions ${ }^{[25]}$. Antioxidant and phase II enzyme (quinine reductase)-inducing activities of beetroot were enriched in regulate and high-pigment red phenotypes compared to orange and white phenotypes ${ }^{[26]}$. Antioxidants, which in many cases are free radical scavengers or quenchers of activated states, comprise a vast number of classes of organic molecules including most prominently the phenolics. About $0.5-1 \%$ of extracted beet juice solids are betalains. In addition to the pigments, the root contains about 700-800 mg oxalic acid and 5-6 mg ascorbic acid per $100 \mathrm{~g}$ beetroot ${ }^{[27]}$.

The increased production of oxidants and free radicals during inflammatory disorders has become widely recognised as integral component of cell and tissue injury ${ }^{[4]}$. Interferon- $\gamma$ potently induces production and release of ROS in macrophages ${ }^{[8]}$. All inflammatory processes include oxygen-activating processes where ROS are produced. Thus, ingredients of beetroot, like phenols and ascorbic acid ${ }^{[22,27]}$ may detoxify ROS acting as chemical antioxidants, moreover, by reducing effects mediated by interferon- $\gamma$, they may also reduce the formation of ROS. 
Since long activated macrophages have been claimed to boost tumor growth and spread ${ }^{[28]}$. Only today pathways by which these cells may support tumor development are better defined and nuclear transcription factor NF- $\mathrm{KB}$ appears to be a key regulator of them ${ }^{[29]}$. Transcription factors of the NF- $\mathrm{KB}$ family are essential regulators of the inflammatory and immune responses. Radicals directly modulate signalling cascades by activating or inhibiting e.g., NF$\mathrm{KB}$, or more indirectly affecting such signalling by changing the cellular redox status ${ }^{[30]}$.

\section{CONCLUSION}

In conclusion, our in vitro study demonstrates that red beetroot juice interfere with immunologic pathways which involve Th-1 type cytokine IFN- $\gamma$. Data show that compounds have immunosuppressive and antiinflammatory activity. This finding may relate to the health beneficial effects of beetroots.

\section{ACKNOWLEDGEMENT}

This study was supported by the Austrian Federal Ministry of Social Affairs and Generations and by the „Austrian Cancer Society/Tyrol“. The authors thank Miss Astrid Haara for excellent technical assistance.

\section{REFERENCES}

1. Kapadia, G.J., H. Tokuda, T. Konoshima and H. Nishino, 1996. Chemoprevention of lung and skin cancer by Beta vulgaris (beet) root extract. Cancer Lett., 100: 211-214.

2. Rauha, J.P., S. Remes, M. Heinonen, A. Hopia and M. Kahkonen et al., 2000. Antimicrobial effects of Finnish plant extracts containing flavonoids and other phenolic compounds. Intl. J. Food Microbiol., 25: 3-12.

3. Prahoveanu, E., V. Esanu, G. Anton and S. Frunzulica, 1986. Prophylactic effect of a Beta vulgaris extract on experimental influenza infection in mice. Virologie, 37: 121-123.

4. Gutteridge, J.M. and B. Halliwell, 2000. Free radicals and antioxidants in the year 2000. A historical look to the future. Ann. N. Y. Acad. Sci., 899: 136-47.

5. Fortuno, A., G. San Jose, M.U. Moreno, J. Diez and G. Zalba, 2005. Oxidative stress and vascular remodeling. Exp. Physiol., 90: 457-462.

6. Murr, C., B. Widner, B., B. Wirleitner, P. and D. Fuchs, 2000. Neopterin as a marker for immune system activation. Curr. Drug. Metabol., 3: 175-187.

7. Wirleitner, B., G., Neurauter, K., Schröcksnadel, B. Frick and D. Fuchs, 2003. Interferon- $\gamma$-induced conversion of tryptophan: Immunologic and neuropsychiatric aspects. Curr. Med. Chem., 10: $1581-1591$.
8. Nathan, C.F., 1986. Peroxide and pteridine: A hypothesis on the regulation of macrophage antimicrobial activity by interferon gamma. Interferon, 7: 125-143.

9. Reibnegger, G., D. Fuchs, L.C. Fuith, A. Hausen and E.R. Werner et al., 1991. Neopterin as a marker for activated cell-mediated immunity: Application in malignant disease. Cancer Detect. Prevent., 15: 483-490.

10. Murr, C., A. Bergant, M. Widschwendter, K. Heim, H., Schröcksnadel and D. Fuchs, 1999. Neopterin is an independent prognostic parameter in females with breast cancer. Clin. Chem., 45: 1998-2004.

11. Giusti, R.M., E.M. Maloney, B. Hanchard, O.S.C. Morgan and S.M. Steinberg et al., 1996. Differential patterns of serum biomarkers of immune activation in human T-cell lymphotropic virus type I-associated myelopathy/tropical spastic pararesis and adult T-cell leukemia/lymphoma. Cancer Epidemiol. Biomarkers Prevent., 5: 699-709.

12. Huang, A., D. Fuchs, B. Widner, C. Glover, D.C. Henderson and T.G. Allen-Mersh, 2002. Serum tryptophan decrease correlates with immune activation and impaired quality of life in colorectal cancer. Brit. J. Cancer, 86: 1691-1696.

13. Winkler, C., B. Wirleitner, K. Schroecksnadel, H. Schennach, E. Mur and D. Fuchs, 2004. In vitro effects of two extracts and two pure alkaloid preparations of Uncaria tomentosa on peripheral blood mononuclear cells. Planta Med., 70: 205-210.

14. Winkler, C., B. Wirleitner, K. Schroecksnadel, H. Schennach and D. Fuchs, 2004. St. John's wort (Hypericum perforatum) counteracts cytokineinduced tryptophan catabolism in vitro. Biol. Chem., 385: 1197-1202.

15. Widner, B., E.R. Werner, H. Schennach, H. Wachter and D. Fuchs, 1997. Simultaneous measurement of serum tryptophan and kynurenine by HPLC. Clin. Chem., 43: 2424-2426.

16. Huber, C., J.R. Batchelor, D. Fuchs, A. Hausen and A. Lang et al., 1984. Immune response-associated production of neopterin-release from macrophages primarily under control of interferon-gamma. J. Exp. Med., 160: 310-316.

17. Byrne, G.I., L.K. Lehmann, J.G. Kirschbaum, E.C. Borden, C.M. Lee and R.R. Brown, 1986. Induction of tryptophan degradation in vitro and in vivo: A gamma-interferon-stimulated activity. J. Interferon Res., 6: 389-396.

18. Weiss, G., C. Murr, H. Zoller, M. Haun, B. Widner, C. Ludescher and D. Fuchs, 1999. Modulation of neopterin formation and tryptophan degradation by Th1-and Th2-derived cytokines in human monocytic cells. Clin. Exp. Immunol., 116: 435-440. 
19. Zvetkova, E., B. Wirleitner, NT. Tram, H. Schennach and D. Fuchs, 2001. Aqueous extracts of Crinum latifolium (L.) and Camellia sinensis show immunomodulatory properties in human peripheral blood mononuclear cells. Intl. Immunopharmacol., 1: 2143-50.

20. Wirleitner B., K. Schroecksnadel, C. Winkler, H. Schennach and D. Fuchs, 2005. Resveratrol suppresses interferon-gamma-induced biochemical pathways in human peripheral blood mononuclear cells in vitro Immunol. Lett., 100: 159-163.

21. Murr, C., K. Schroecksnadel, C. Winkler, M. Ledochowski and D. Fuchs, 2005. Antioxidants may increase the probability of developing allergic diseases and asthma. Med. Hypotheses, 64: 973-977.

22. Vinson, J.A., Y. Hao, C. Su and L. Zubic, 1998. Phenol antioxidant quantity and quality in foods: vegetables. J. Agric. Food Chem., 46: 3630-3634.

23. Kähkönen, M.P., A.I. Hopia, H.J. Vuorela, J.P. Rauha and K. Pihlaja et al., 1999. Antioxidant activity of plant extracts containing phenolic compounds. J. Agric. Food Chem., 47: 3954-3962.

24. Kujala, T.S., J.M. Loponen, K.D. Klika and K. Pihlaja, 2000. Phenolics and Betacyanins in red beetroot (Beta vulgaris) root: Distribution and effect of cold storage on the content of total phenolics and three individual compounds. J. Agric. Food Chem., 48: 5338-5342.

25. Escribano, J., M.A. Pedreno, F. Garcia-Carmona and R. Munoz, 1998. Characterization of the antiradical activity of betalains from Beta vulgaris L. roots. Phytochem. Anal., 9: 124-127.
26. Wettasinghe, M., B. Bolling, L. Plhak, H. Xiao and K. Parkin. 2002. Phase II enzyme-inducing antioxidant activities of beetroot (Beta vulgaris L.) extracts from phenotypes of different pigmentation. J. Agric. Food Chem., 50: 6704-6709.

27. Eastwood, M.A. and H. Nyhlin, 1995. Beeturia and colonic oxalic acid. Q. J. Med., 88: 711-717.

28. Munzarova, M. and J. Kovarik, 1987. Is cancer a macrophage-mediated autoaggressive disease? Lancet., 1: 952-954.

29. Schreck, R., P. Rieber and P.A. Baeuerle, 1991. Reactive oxygen intermediates as apparently widely used messengers in the activation of the NF-kappa B transcription factor and HIV-1. EMBO J., 10: 2247-2258.

30. Li, Q., S. Withoff and I.M. Verma, 2005. Inflammation-associated cancer: NF-kappaB is the lynchpin. Trends Immunol., 26: 318-325. 